\title{
Amelioration of collagen-induced arthritis using antigen-loaded dendritic cells modified with NF- $\kappa B$ decoy oligodeoxynucleotides
}

This article was published in the following Dove Press journal:

Drug Design, Development and Therapy

13 October 2017

Number of times this article has been viewed

\author{
Hongmei Jiang' \\ Henggui $\mathrm{Hu}^{2}$ \\ Yali Zhang' \\ Ping Yue ${ }^{3}$ \\ Lichang Ning' \\ Yan Zhou' \\ Ping Shi' \\ Rui Yuan'
}

'School of Clinical Laboratory Science, Guizhou Medical University, Guiyang, Guizhou, ${ }^{2}$ Department of Clinical Laboratory, The Third Hospital Subsidiary of Bengbu Medical College, Suzhou, Anhui, ${ }^{3}$ School of Biology and Engineering, Guizhou Medical University, Guiyang, Guizhou, People's Republic of China
Correspondence: Hongmei Jiang School of Clinical Laboratory Science, Guizhou Medical University, 9 Beijing Road, Yunyan District, Guiyang, Guizhou 550004, People's Republic of China Tel +86 I3984I2 6067 Email jhmzq1973@163.com

\begin{abstract}
Dendritic cells (DCs) play an important role in the initiation of autoimmunity in rheumatoid arthritis (RA); therefore, the use of DCs needs to be explored to develop new therapeutic approaches for RA. Here, we investigated the therapeutic effect of bovine type II collagen (BIIC)-loaded DCs modified with NF- $\mathrm{KB}$ decoy oligodeoxynucleotides (ODNs) on collageninduced arthritis (CIA) in rats and explored the underlying mechanisms. DCs treated with BIIC and NF- $\kappa \mathrm{B}$ decoy ODNs exhibited features of immature DCs with low levels of costimulatory molecule (CD80 and CD86) expression. The development of arthritis in rats with CIA injected with BIIC + NF- $\kappa$ B decoy ODN-propagated DCs (BIIC-decoy DCs) was significantly ameliorated compared to that in rats injected with BIIC-propagated DCs or phosphate-buffered saline. We also found that the BIIC-decoy DCs exerted antiarthritis effects by inhibiting selflymphocyte proliferative response and suppressing IFN- $\gamma$ and anti-BIIC antibody production and inducing IL-10 antibody production. Additionally, antihuman serum antibodies were successfully produced in the rats treated with BIIC-decoy DCs but not in those treated with NF- $\kappa \mathrm{B}$ decoy ODN-propagated DCs; moreover, the BIIC-decoy DCs did not affect immune function in the normal rats. These findings suggested that NF- $\kappa B$ decoy ODN-modified DCs loaded with a specific antigen might offer a practical method for the treatment of human RA.
\end{abstract}

Keywords: NF- $\mathrm{KB}$ decoy oligodeoxynucleotides, collagen-induced arthritis, dendritic cells, rheumatoid arthritis

\section{Introduction}

Rheumatoid arthritis (RA), a chronic autoimmune disease, is considered one of the important causes of disability. Currently, immunosuppressive drugs are one of the major therapeutic approaches used for RA. However, these drugs can induce a generalized immune suppression that increases the risk of other inflammatory diseases. ${ }^{1}$ Thus, a better therapeutic approach for RA ideally needs to suppress the inflammation and establish tolerance toward arthritogenic antigens without impairing immune system function. $^{2}$

T lymphocytes are one of the most abundant forms of lymphocyte cell types in the RA synovium; accordingly, RA has been reported as a kind of autoimmune disease that is primarily mediated by $\mathrm{T}$ lymphocytes. ${ }^{3,4}$ Moreover, a recent study has found that the abnormal activation, differentiation, and secretion of cytokines in $\mathrm{CD}^{+}$ T cells play an important role in orchestrating RA. ${ }^{5}$ Dendritic cells (DCs), a kind of antigen-presenting cells (APCs) that play a key role in the activation of T cells, are the only APCs known to activate naive T cells. Mature DCs (mDCs) have effective 
immunostimulatory ability characterized by a high expression level of cell surface molecules of major histocompatibility complex class II, and T cells stimulate cytokines, especially IL-12. Nevertheless, immature DCs (imDCs) can enhance antigen-specific tolerance in vivo ${ }^{6}$ and myeloid derivedimDCs are capable of invalidating alloantigen-specific T lymphocytes. $^{7}$ This important role of imDCs in the induction of immune tolerance has already attracted widespread attention. For instance, Figdor et al suggested that imDCs prevent autoimmune reactions by blocking or reducing immune activation and that they can therefore be used for the treatment of transplantation rejection, allergy, autoimmune diseases, and chronic inflammation. ${ }^{8}$ Giannoukakis et al showed that the tolerogenic DCs (tDCs) generated from donors in vitro can prolong a patient's survival time by importing tDCs into the recipient's body. ${ }^{9,10}$ In another study, in a murine collageninduced arthritis (CIA) model, the progression of arthritis was suppressed by concurrent treatment with tDCs and mesenchymal stem cells. ${ }^{11}$ However, so far, there have been no reports on RA treatment using NF- $\kappa \mathrm{B}$ decoy oligodeoxynucleotide (ODN)-modified DCs loaded with a specific antigen.

Recent studies have shown that DC maturation and immunostimulatory response rely on $\mathrm{NF}-\kappa \mathrm{B}$-dependent gene transcription. ${ }^{12}$ Inactivation of NF- $\mathrm{KB}-$ mediated signaling suppresses the maturation and activation of DCs. ${ }^{13}$ Bone marrow-derived DCs treated with NF- $\mathrm{KB}$ decoy (ODNs) have been reported to show immaturity in phenotype and production of induced Th1 cytokines, and these imDCs can cause immune tolerance and reduce graft rejection. ${ }^{9}$ Therefore, using NF- $\mathrm{KB}$ as a therapeutic target for inhibiting or regulating the division of DCs may be a new strategy for inducing immune tolerance. However, it is uncertain whether genetically engineered DCs can induce immune tolerance against RA.

In the present study, we aimed to investigate whether specific antigen-loaded DCs modified with NF- $\kappa$ B decoy ODNs are effective in mediating the progression of CIA in rats.

\section{Materials and methods}

\section{Animals}

Female Sprague Dawley (SD) rats weighing 200-250 g were used in this study. The animals were maintained at $21^{\circ} \mathrm{C} \pm 2{ }^{\circ} \mathrm{C}$ and on a $12 \mathrm{~h}$ light/dark cycle with free access to standard laboratory rat food pellets and water. The research procedures were approved by the Animal Ethics Committee of Guiyang Medical College. All animal experiments were performed in accordance with the Guide for the Care and Use of Laboratory Animals (guide number 1602019).

\section{Synthesis of NF-KB decoy ODNs}

Double-stranded NF- $\kappa \mathrm{B}$ decoy ODNs were generated using equimolar amounts of single-stranded sense and antisense phosphorothioate-modified oligonucleotides containing two NF- $\kappa \mathrm{B}$ binding sites (sense sequence $5^{\prime}$-A GGGACTTTCCGC TGGGGACTTTCC- $3^{\prime}$, the nucleotides underlined indicate the

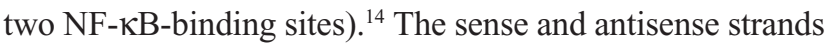
of each oligonucleotide were mixed in $150 \mathrm{mM}$ phosphatebuffered saline (PBS), heated to $100^{\circ} \mathrm{C}$, and allowed to cool to room temperature to obtain double-stranded DNA.

\section{Isolation of mononuclear cells from the spleen}

Rats were anesthetized via intraperitoneal injection of $10 \%$ chloral hydrate $(0.3-0.4 \mathrm{~mL} / 100 \mathrm{~g})$. The spleen tissue was then excised and placed in $5 \mathrm{~mL}$ RPMI-1640 incomplete medium and stored at $4{ }^{\circ} \mathrm{C}$ until use. The splenic tissues were washed with PBS, and $3 \mathrm{~mL}$ collagenase IV digestion liquid was added; the tissue specimens were then cut into pieces, followed by incubation at $37^{\circ} \mathrm{C}$ for $30 \mathrm{~min}$. After grinding and filtering through a 200 mesh screen, the cell suspension was collected into tubes and centrifuged at 1,500 rpm for $10 \mathrm{~min}$. The cell pellet was then washed with PBS two times, suspended in $3 \mathrm{~mL} 2 \%$ newborn bovine serum, and stacked on the surface of $3 \mathrm{~mL}$ of rat mononuclear cell separation fluid. This solution was then again centrifuged at 2,000 rpm for $20 \mathrm{~min}$ and the resulting single nucleus cell layer was absorbed and washed with PBS. Finally, the cells were resuspended in RPMI-1640 incomplete medium and adjusted to obtain a cell density of $5 \times 10^{7}$ cells $/ \mathrm{mL}$. Next, $2-3 \mathrm{~mL}$ of this cell suspension was transferred onto six-well plates and cultured at $37^{\circ} \mathrm{C}$ under $5 \% \mathrm{CO}_{2}$ for $90 \mathrm{~min}$. Finally, the culture medium was gently removed and the cells were washed with preheated PBS; the cells that did not adhere to the walls were removed.

\section{Propagation of spleen-derived DC populations}

The mononuclear cells harvested from the spleens of SD rats were initially plated and cultured in 24-well plates $\left(2 \times 10^{6}\right.$ cells/well) in $2 \mathrm{~mL}$ of RPMI- 1640 complete medium for $2 \mathrm{~h}$. After the removal of unattached cells, the remaining cells were further cultured in RPMI-1640 medium supplemented with antibiotics, $10 \%$ fetal calf serum, $10 \mathrm{ng} / \mathrm{mL}$ recombinant rat granulocyte-macrophage colony-stimulating factor, and $10 \mathrm{ng} / \mathrm{mL}$ recombinant rat IL-4 to induce their development into $\mathrm{mDCs}$. At the point of culture initiation, $10 \mu \mathrm{mol} / \mathrm{L} \mathrm{NF}-\kappa B$ decoy ODNs or vehicle were included in 
some wells under the following four conditions: 1) control group, DCs were treated with cytokines only; 2) lipopolysaccharide (LPS) group, DCs were treated with $10 \mu \mathrm{g} / \mathrm{mL}$ LPS on the sixth day of culture; 3 ) decoy group, DCs were treated with $10 \mu \mathrm{mol} / \mathrm{L} \mathrm{NF- \kappa B}$ decoy ODNs; 4) bovine type II collagen + NF- $\kappa$ B decoy ODN-propagated DCs (BIIC-decoy DCs) group, DCs were treated with $10 \mu \mathrm{mol} / \mathrm{L} \mathrm{NF- \kappa B}$ decoy ODNs, followed by treatment with $50 \mu \mathrm{g} / \mathrm{mL}$ bovine type II collagen (BIIC) on the fourth day. The cytokine-enriched medium was refreshed every 3 days. On the seventh day of culture, the nonadherent DCs released spontaneously from the clusters were harvested.

\section{Fluorescence-activated cell sorting (FACS)}

The degree of purity and maturity of the DCs was assessed by the expression of OX-62, CD80, and CD86 using FACS. Briefly, the cells were separately collected into FACS tubes and diluted $1 \times 10^{6}$ cells/tube. Two milliliters of PBS was then added to each tube, followed by centrifugation at 1,000 rpm for $10 \mathrm{~min}$. Next, the supernatant was removed and $100 \mu \mathrm{L}$ PBS was added to resuspend the cells to obtain a single cell suspension. Next, PE-OX-62 (cat \#: 12-1030-80, lot \#: E032645, clone OX-62; eBioscience, San Diego, CA, USA), PE-CD80 (cat \#: 12-0800-81, lot \#: E034706, clone 3H5; eBioscience), and FITC-CD86 (cat \#: 11-0860-81, lot \#: E027724, clone 24F; eBioscience) were added at final concentrations of $1.5 \mu \mathrm{L} / 10^{6}$ cells, $2 \mu \mathrm{L} / 10^{6}$ cells, and $0.8 \mu \mathrm{L} / 10^{6}$ cells, respectively, and incubated for $40 \mathrm{~min}$. OX-62 was detected by single labeling, while CD80 and CD86 were detected using double-labeling flow cytometry. After the reaction, $1 \mathrm{~mL}$ PBS was added to the cells, followed by centrifugation at 1,000 rpm for $10 \mathrm{~min}$; this process was repeated twice. Finally, $100 \mu \mathrm{L}$ PBS was added to resuspend the cells and the resulting suspension was analyzed using FACS.

\section{Primary mixed lymphocyte reaction (MLR) analysis}

MLR was analyzed using the cultured DCs (control DCs, LPS DCs, BIIC-decoy DCs, BIIC-decoy DCs with human serum or BIIC, or BIIC-decoy DCs with BIIC) as the stimulator cells and $\mathrm{T}$ lymphocytes from female Wistar rats as the responder cells. Briefly, the spleen tissue was obtained from female Wistar rats and single-cell suspensions were obtained by glass grinding. The cells were then washed and resuspended in $2 \%$ newborn bovine serum to a final volume of $3 \mathrm{~mL}$; this suspension was then carefully added on the surface of $3 \mathrm{~mL}$ of lymphocyte separation medium and centrifuged at 2,000 rpm for $20 \mathrm{~min}$. The middle layer of cells was then carefully absorbed with pipette and resuspended with $3 \mathrm{~mL}$ d-Hanks liquid and then centrifuged at 2,000 rpm for $10 \mathrm{~min}$. Next, the supernatant was removed, and the cells were again suspended in $4 \mathrm{~mL}$ d-Hanks liquid and centrifuged at $600 \mathrm{rpm}$ for 10 min, followed by washing two times with PBS. Finally, the cells were resuspended in a RPMI-1640 complete medium, adjusted to a concentration of $1 \times 10^{6}$ cells $/ \mathrm{mL}$ and used as the reaction cells. Additionally, total lymphocyte was used in MLR. T cells were plated in a 96-well plate at a density of $5 \times 10^{5}$ cells/well. The stimulator DCs were added and co-cultured with the responder cells at a ratio of 1:5 in complete RPMI-1640 medium. After $68 \mathrm{~h}$ of incubation at $37^{\circ} \mathrm{C}$ under $5 \% \mathrm{CO}_{2}$, the samples were centrifuged at $600 \mathrm{rpm}$ for $10 \mathrm{~min}$, and $50 \mu \mathrm{L}$ of supernatant from each well was gently transferred into clean epoxy tubes, followed by grouping and storage at $-20^{\circ} \mathrm{C}$. MTT (3-(4,5)-dimethylthiahiazo (-z-y1)3,5-di-phenytetrazoliumromide) was dissolved in sterilized PBS by $5 \mathrm{mg} / \mathrm{mL}$ and was filtered through a $0.2 \mu \mathrm{m}$ filter. Next, $20 \mu \mathrm{L}$ MTT was added to each well and the incubation was continued at $37^{\circ} \mathrm{C}$ for $4 \mathrm{~h}$ until cells with a purple dot grain appeared under the microscope. After the removal of culture medium, $100 \mu \mathrm{L}$ dimethyl sulfoxide was added to dissolve the MTT products for $10 \mathrm{~min}$, followed by absorbance measurement at $490 \mathrm{~nm}$ using a microplate reader.

\section{Induction and assessment of $\mathrm{CIA}$ in rats}

We established the animal model of CIA using the same protocol as previously described. ${ }^{12}$ Lyophilized BIIC (SigmaAldrich Co., St Louis, MO, USA) was dissolved at a concentration of $2 \mathrm{mg} / \mathrm{mL}$ in $0.01 \mathrm{~mol} / \mathrm{L}$ acetic acid and kept overnight at $4^{\circ} \mathrm{C}$. The BIIC emulsion was prepared by mixing and emulsifying complete Freund's adjuvant and an equal volume of BIIC. Eight-week-old female SD rats were subcutaneously immunized at the right foot plantar with $200 \mu \mathrm{L}$ BIIC emulsion and subcutaneously immunized with an equal volume of BIIC at the dorsum after 14 days. The onset of CIA usually initiated between days 11 and 13 after the first immunization. The severity of arthritis developed in each hind paw of the CIA rats was scored and recorded as an averaged score obtained by four experimental researchers who assessed the arthritis independently and blindly on a 5-point scale: 0 , no redness or swelling; 1, slight swelling in the ankle or redness in the foot; 2, progressive swelling, inflammation, and redness from the ankle to the midfoot; 3 , swelling and inflammation of the entire foot, not including the toes; 4, swelling and inflammation of the entire foot, including the toes; and 5, swelling and inflammation of the entire foot, with loss of mobility. 


\section{Treatment of $\mathrm{CIA}$ rats with $\mathrm{DCs}$}

On the fifth day after the first immunization, the CIA rats were injected via the tail vein with cultured DCs $\left(5 \times 10^{6} / 0.5 \mathrm{~mL}\right)$ prepared as described earlier. The rats in the experimental group were randomly divided into six groups with the following treatments: 1) BIIC-decoy DCs group ( $\mathrm{n}=10)$ : CIA rats injected with NF-KB decoy ODNs (Sangon, Shanghai, China) + BIIC-pretreated DCs; 2) decoy DCs group $(n=10)$ : CIA rats injected with NF- $\mathrm{KB}$ decoy ODNmodified DCs; 3) BIIC DCs model group ( $\mathrm{n}=10)$ : CIA rats injected with BIIC DCs; 4) CIA model group (n=5): CIA rats injected with PBS; 5) BIIC-decoy DCs control group $(n=5)$ : normal rats injected with NF- $\mathrm{KB}$ decoy ODNs + BIIC-pretreated DCs; and 6) control group $(n=5)$ : normal rats injected with PBS. Twenty days after the initial immunization, human sera were injected into the rats in the BIIC DCs model group, decoy DCs experimental group, and BIIC-decoy DCs experimental group by subcutaneous immunization. The treatment lasted for 42 days. On the final day of treatment, all the rats were anesthetized with $10 \%$ chloral hydrate and blood specimens were collected from each rat by phlebotomy.

\section{Histopathological analysis}

Histopathology analysis was performed on the sections of the paw joints. Briefly, the paw joints were excised from the hind limbs of the rats after removal of the skin, superficial muscle, and tissue, followed by fixation in $4 \%$ polyoxymethylene solution at $4{ }^{\circ} \mathrm{C}$ for $48 \mathrm{~h}$ and decalcification in $10 \%$ ethylene diamine tetraacetic acid-2Na for 35 days at $4{ }^{\circ} \mathrm{C}$. The joints were then dehydrated with alcohol, embedded in paraffin, sectioned into 3-4 $\mu \mathrm{m}$ thick slices, and placed on polylysinecoated slides, followed by staining with hematoxylin and eosin. The histological grading of the articular synovium was scored as follows: for the joint surface, 0 points: smooth, 1 point: a small amount of local rough, 2 points: easy to see rough, and 3 points: more obvious rough; for synovial hyperplasia, 0 points: two to three layers of synovial cell in alignment, with no hyperplasia, 1 point: a small number of synovial cells with hyperplasia and permutation, 2 points: the number of synovial cell layers was obviously increased and the synovial cells were disordered, and 3 points: the synovial membrane was obviously hyperplasic and the fluff and pannus were formed; and for inflammatory cells, 0 points: no infiltrated cells, 1 point: a small number of inflammatory cells, 2 points: dense and infiltrated inflammatory cells were visible, and 3 points: a large number of inflammatory cells were infiltrated.
Measurements of IL-I0, IFN- $\gamma$, anti-BIIC, and anti-human serum antibody levels

The levels of cytokines (including IL-10 and IFN- $\gamma$ ) and the immunoglobulin $\mathrm{G}$ level of anti-BIIC in the rat sera were measured using two types of enzyme-linked immunosorbent assay kits (Ameko, Shanghai, China) in accordance with the manufacturer's instructions. The antibody titers against human serum in the rats were measured by double immunodiffusion assay.

\section{Secondary MLR analysis}

Secondary MLR was performed to explore whether the DCs stimulated antigen specificity of T-cell response. DCs isolated from the spleen of the CIA rats were used as the stimulator cells. T cells isolated from the spleens of female Wistar rats were used as the responder cells. DCs or T cells alone that were incubated in the medium served as the stimulator controls and responder controls, respectively. The experimental procedures used to incubate and harvest cells were similar to those described earlier.

\section{Statistical analysis}

Statistical analysis of data was performed using the Student's $t$-test and rank sum test. A value of $P<0.05$ was considered statistically significant.

\section{Results}

\section{Purity identification of spleen-derived DCs}

To identify the purity of the rat spleen-derived DCs, the expression ratio of the DC-specific marker OX-62 was measured using FACS. As expected, the expression ratio of OX-62 was $>72.6 \%$ in each group (Figure 1A), which suggested that the DCs obtained for this study were quite high in purity.

\section{NF-KB decoy ODNs inhibited DC maturation}

Functional maturation of the DCs is related to upregulation of costimulatory surface molecules (eg, CD80 and CD86). Therefore, to assess the ability of NF- $\mathrm{KB}$ decoy ODNs to suppress DC maturation, DCs were treated with NF- $\mathrm{KB}$ decoy ODNs or NF- $\kappa$ B decoy ODNs + BIIC. FACS analysis showed that the control DCs and LPS DCs exhibited mature phenotypical features with high expression of CD80 and CD86 (Figure 1A and B). However, the decoy DCs, LPSdecoy DCs, and BIIC-decoy DCs displayed an immature state with lower expression of CD80 and CD86 compared to the control and LPS DCs. 

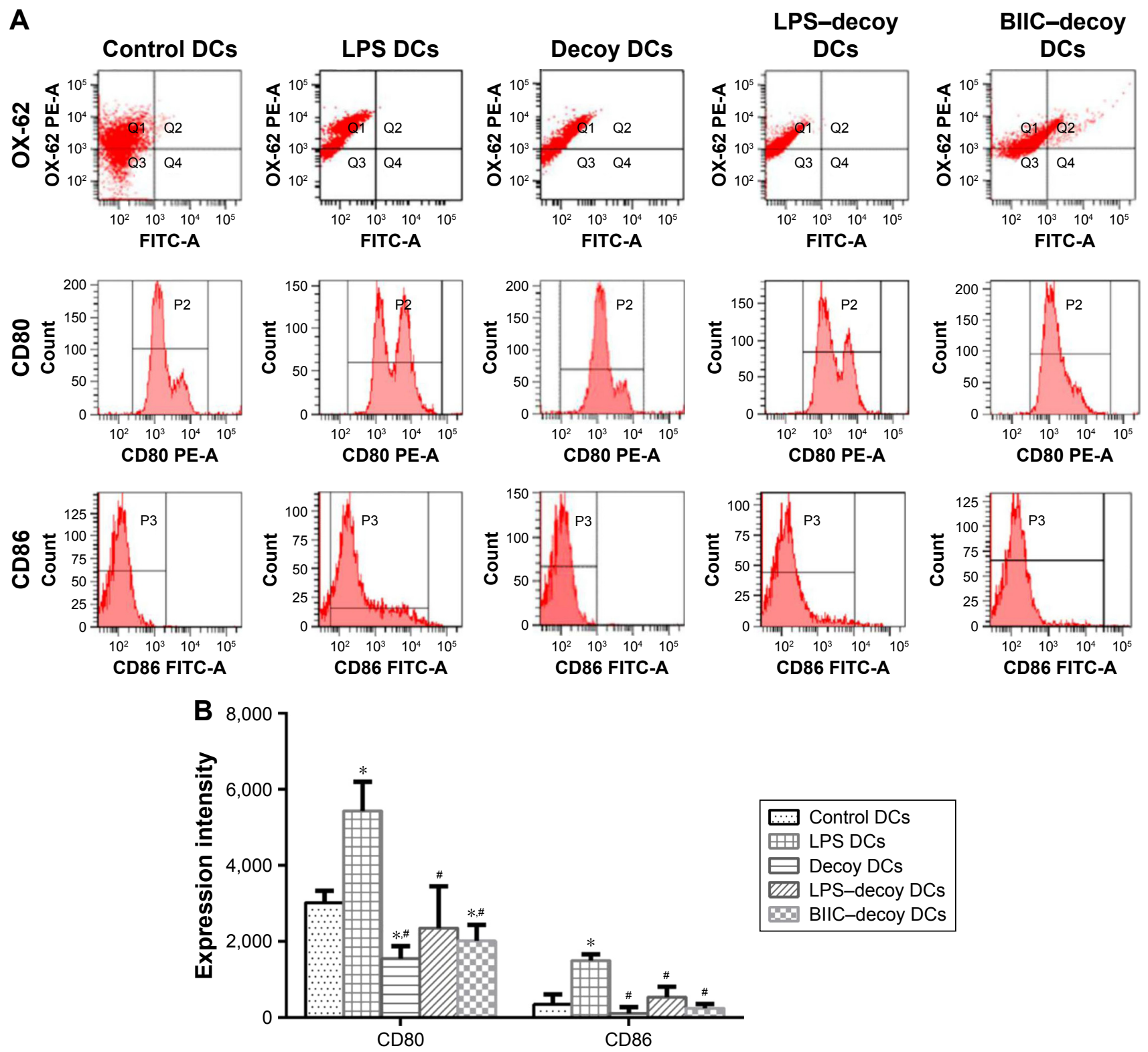

Figure I Effect of LPS, NF-KB decoy ODN, and BIIC-decoy on the expression of OX-62, CD80, and CD86 on the DCs.

Notes: (A) Flow cytometric point diagram analysis of OX-62 and histogram analysis of CD80 and CD86 on DCs. The demarcation of CD80 CD86 MFI was scribed according to the blank control group. (B) Quantitative analysis of CD80 and CD86 expression on treated DCs. $* P<0.05$ vs control, ${ }^{*} P<0.05$ vs LPS DCs.

Abbreviations: BIIC, bovine type II collagen; DCs, dendritic cells; LPS, lipopolysaccharide; MFI, mean fluorescence intensity; ODN, oligodeoxynucleotide.

\section{NF-KB decoy ODNs inhibited primary MLR}

To study the effect of NF- $\mathrm{KB}$ decoy ODNs on the antigen presenting ability of DCs and their vital role in immune activation, we assessed the influence of decoy DCs on lymphocyte proliferation capability. In MLR, the proliferation ability of T lymphocytes of the decoy group was significantly lower than that of the control and LPS groups $(P<0.05)$; the proliferation ability increased after LPS addition but was still lower than that of the LPS group (Figure 2). Additionally, addition of human serum significantly increased the stimulation capability of DCs treated with BIIC and NF- $\mathrm{\kappa B}$ decoy ODNs.

\section{BIIC-decoy DCs ameliorated joint swelling and arthritis in CIA rats}

One of the characteristics of the initiation and development of RA is the presence of inflammatory joints. Several hours postinjection of PBS into the control rats, the injected foot showed obvious swelling but returned to normal after 3 days and no recurrence was observed (Figure 3A). However, in the CIA group immunized with BIIC, the hind paws all 


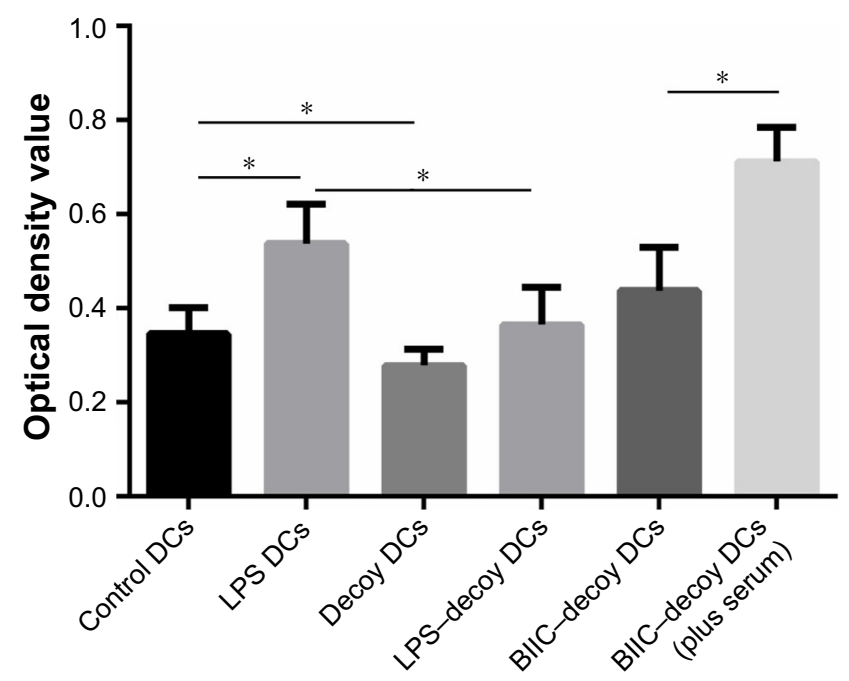

Figure 2 Influence of LPS, NF- $\kappa B$ decoy ODN, and BIIC-decoy on the stimulation capability of DCs in MLR.

Notes: $\mathrm{n}=10 ; * \mathrm{p}<0.05$.

Abbreviations: BIIC, bovine type II collagen; DCs, dendritic cells; LPS, lipopolysaccharide; MLR, mixed lymphocyte reaction; ODN, oligodeoxynucleotide.

presented with erythema and swelling on the third day; after boosting with BIIC, erythema and severe swelling encompassed the entire ankle, foot, and digits, and some rats even appeared ulcerate in the left hind metatarsal footpad, which suggested that BIIC effectively induced human-like RA in the rats (Figure 3B).

To investigate the therapeutic effects of the modified DCs on CIA rats, the CIA rats were treated with BIIC DCs, decoy DCs, or BIIC-decoy DCs. We found that the joint swelling of rats in the BIIC DCs group (Figure 3C) was more serious than that in the CIA group. However, the hind paws of CIA rats treated with BIIC-decoy DCs (Figure 3D) or decoy DCs (Figure 3E) were significantly less prone to develop erythema and swelling compared to those of the untreated or BIIC DCs treated CIA rats. Additionally, after treatment for 21 days, the arthritis index of the BIIC-decoy DCs and decoy DCs groups was significantly lower than that of the CIA and BIIC DCs groups $(P<0.01)$ and these therapeutic effects lasted for at least 42 days (Figure 3F). Moreover, there was no significant difference in the therapeutic effects between the BIIC-decoy DCs and decoy DCs.

\section{BIIC-decoy DCs mitigated}

\section{pathomorphological change in $\mathrm{CIA}$ rats}

Histological analysis showed that the articular synovial tissue of the rats in the control group was composed of two to three layers of synovial cells and synovial subintima, with neat rows of synovial membrane cells; the joint surface was smooth, and there was no inflammatory cell infiltration in the joint cavity (Figure 4A). In contrast, in the CIA and BIIC
DCs groups, the articular surfaces in the arthritis joints of rats were not smooth, with a number of inflammatory cells infiltrated into the synovial membranes, which was accompanied by synovial hyperplasia and pannus formation (Figure 4B and C). However, the BIIC-decoy DCs and decoy DCs not only ameliorated the infiltration of the inflammatory cells into the joint cavity but also significantly decreased the synovial hyperplasia and pannus formation (Figure 4D and E). The histological scores of the articular synovium in the BIIC-decoy DCs and decoy DCs groups were significantly decreased compared to those in the CIA and BIIC DCs groups (Figure 4F).

\section{BIIC-decoy DCs modulated ThI- and Th2-type-related cytokine release}

After treatment for 5 weeks, the serum levels of anti-BIIC antibody and IFN- $\gamma$ in the CIA and BIIC DCs groups were significantly higher than those in the control rats $(P<0.01)$ (Figure 5). Moreover, compared to the CIA and BIIC DCs groups, the BIIC-decoy DCs and decoy DCs groups expressed significantly lower levels of anti-BIIC antibody and IFN- $\gamma(P<0.01)$. In contrast, the serum levels of IL-10 in the CIA and BIIC DCs groups were significantly lower than those in the control group $(P<0.01)$, but the serum levels of IL-10 in the BIIC-decoy DCs and decoy DCs groups were significantly higher than those in the CIA or BIIC DCs groups $(P<0.01)$. Furthermore, all the cytokine levels in the rats treated with BIIC-decoy DCs and decoy DCs were indistinguishable. Thus, BIIC-decoy DCs showed a distinct feature of being able to regulate cytokine release and antibody production from Th1 and Th2 cells.

\section{BIIC-decoy DCs did not affect immune function in the normal rats}

To survey whether BIIC-decoy DCs affect systemic immune function, we measured the serum levels of IFN- $\gamma$, IL-10, and anti-BIIC antibody immunoglobulin $\mathrm{G}$ in the normal rats treated with BIIC-decoy DCs and found that the levels of these cytokines or antibody were unaltered (Figure 5). This result indicated that BIIC-decoy DCs did not elicit any behavioral or toxic impairment to the normal immune function of the rats.

\section{BIIC-decoy DCs did not affect independent antigen immune response}

To examine the effects of BIIC-decoy DCs on independent antigen immune response, human serum was injected into the rats 20 days after the initial immunization. The serum antibody level against the independent human antigen in the BIIC-decoy DCs group was significantly higher than that in 
A

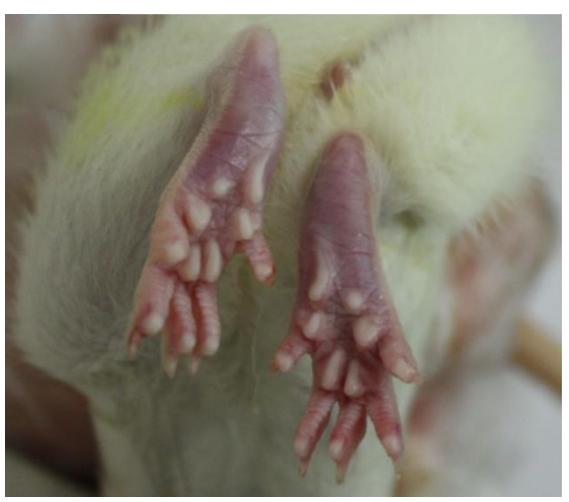

C

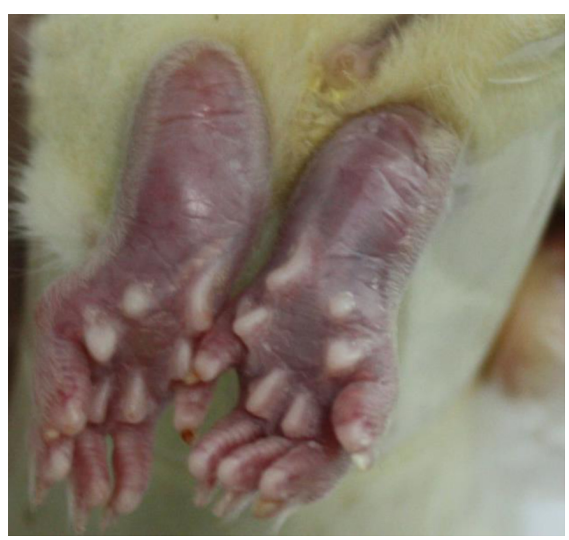

E

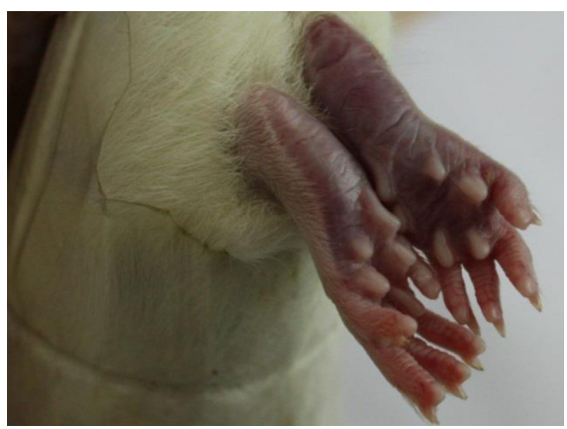

B

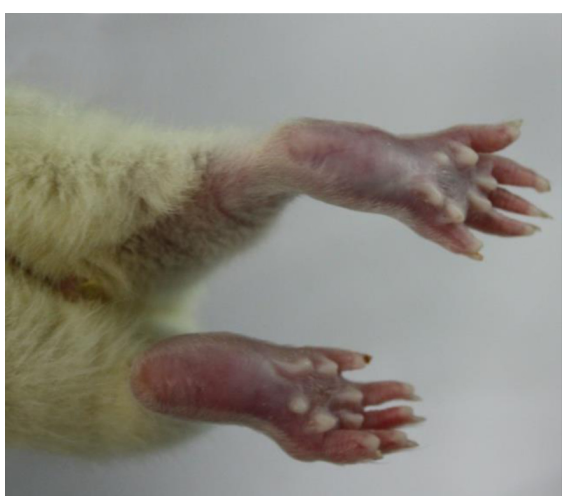

D

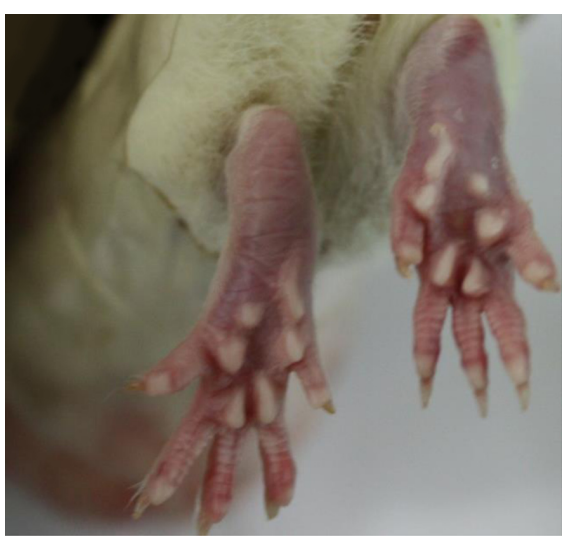

$\mathbf{F}$

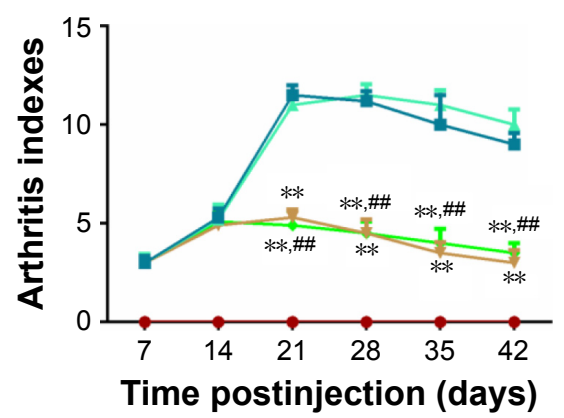

$\begin{array}{ll}\rightarrow-\text { Control group } & - \text { CIA model group } \\ \leftarrow \text { BIIC DCs model group } & - \text { - Decoy DCs group } \\ \leftarrow \text { BIIC-decoy DCs group } & \end{array}$

Figure 3 Severity of arthritis in rats during 42 days posttreatment.

Notes: (A) Control group: normal rats receiving PBS. (B) CIA model group. (C) BIIC DCs model group. (D) Decoy DCs group. (E) BIIC-decoy DCs group. (F) The arthritis indexes of the rats in each group. ${ }^{*} P<0.0$ I vs $C I A$ model group, ${ }^{\#}<0.01$ vs $B I I C D C$ model group.

Abbreviations: BIIC, bovine type II collagen; CIA, collagen-induced arthritis; DCs, dendritic cells; PBS, phosphate-buffered saline.

the BIIC DCs group, but the decoy DCs group failed to produce antibodies (Table 1). Thus, we concluded that the BIIC-decoy DCs did not alter independent antigen immune response.

\section{BIIC-decoy DCs inhibited lymphocyte proliferation}

Compared to the control group, the CIA group showed significantly higher lymphocyte proliferation $(P<0.05)$. However, the BIIC-decoy DCs significantly reduced
CIA-associated lymphocyte proliferation. Provoked by the independent human serum immunization, the stimulated lymphocyte proliferation was also prevented by the BIICdecoy DCs (Figure 6).

\section{Discussion}

The rat model of CIA is the most widely used model to investigate the pathogenesis of RA. Its clinical manifestations, pathological features, immunological changes, and pathogenesis are similar to those of human RA. ${ }^{15}$ In this 
A

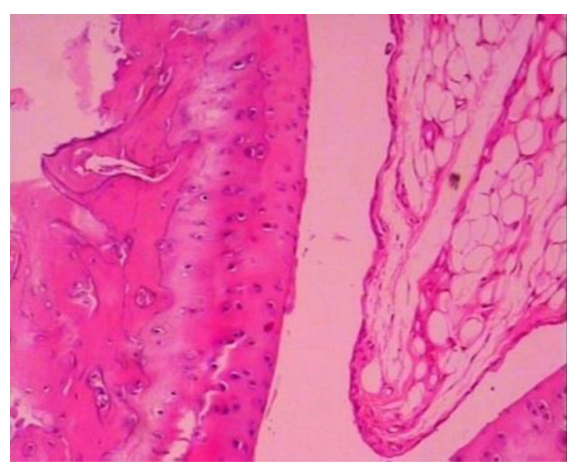

C

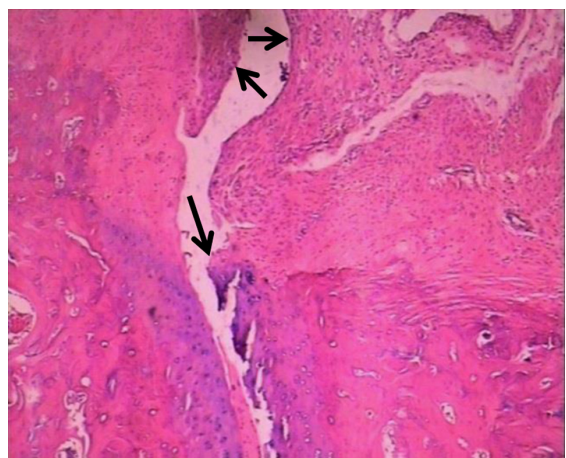

E

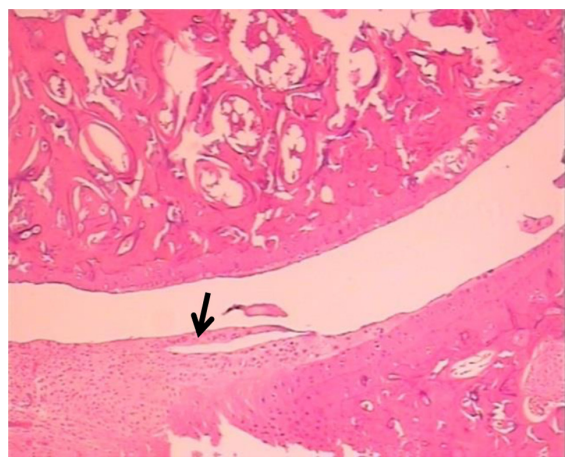

B

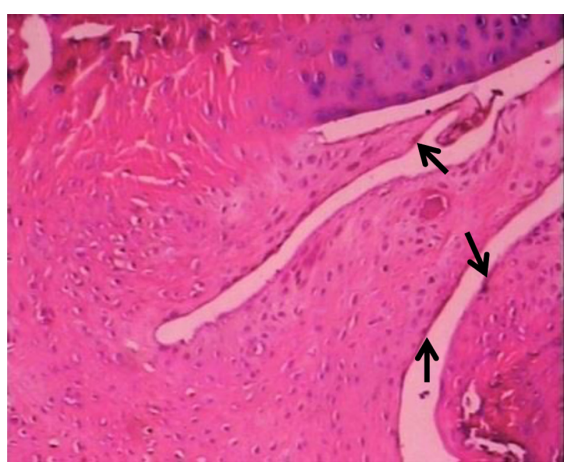

D

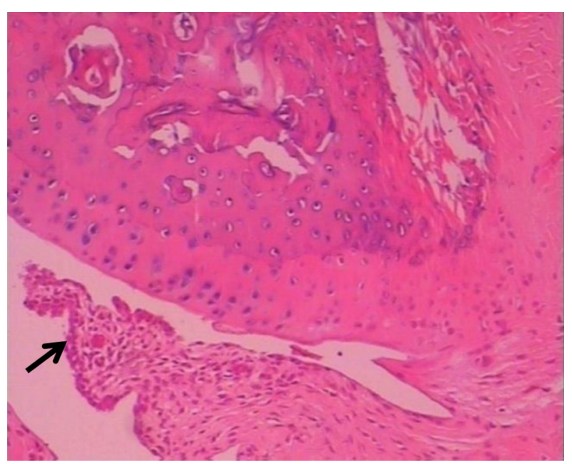

$F \div \widehat{0}$

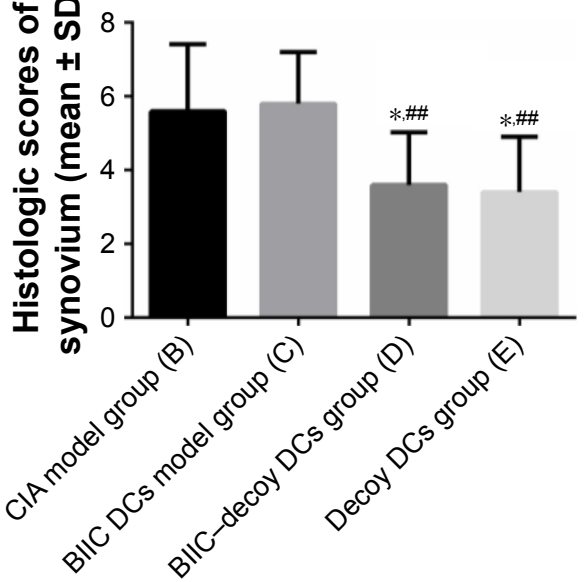

Figure 4 Histological characteristics of ankle joints in the rats at 42 days posttreatment.

Notes: (A) Control group. (B) CIA model group. (C) BIIC DCs model group. (D) Decoy DCs group. (E) BIIC-decoy DCs group. The black arrows indicate the pannus formation sites where the synovial cell proliferation and inflammatory cell infiltration can be found. Magnification $\times 100$. (F) The histological scores of the articular synovium. $* P<0.05$ vs $\mathrm{ClA}$ model group, ${ }^{\# P}<0.0$ I vs BIIC DCs model group.

Abbreviations: BIIC, bovine type II collagen; CIA, collagen-induced arthritis; DCs, dendritic cells.

study, we induced CIA by immunizing SD rats with BIIC. Consistent with a previous report, ${ }^{16}$ the CIA rats in our study showed significantly higher arthritis index than the normal rats and the histological signs were characterized by extensive inflammatory cells infiltration. Moreover, the CIA rats showed similar symptoms, histopathological manifestations, and high level of anti-BIIC antibody as those observed in human RA, which reconfirmed that the CIA rats were ideal animal models for RA study.

Human RA is initiated when the pathogenic antigen is recognized by $\mathrm{T}$ cells, and the abnormal activation and differentiation of T cells play an important role in RA. ${ }^{3}$ DCs play an important role in autoimmunity response and transplantation immunity response and are the only known APCs that can activate initial $\mathrm{T}$ cells. Moreover, mDCs mainly activate immune responses, whereas imDCs can induce immune tolerance. Previous research suggests that the activation of $\mathrm{NF}-\mathrm{KB}$ is a core factor of DC maturity, because of which most of NF- $\mathrm{KB}$ activators can induce DC maturity. Additionally, activated NF- $\kappa B$ plays a key role in the activation of $\mathrm{T}$ cells by DCs and its continuous activation strengthens this effect. ${ }^{17}$ NF- $\kappa B$ decoy ODNs have a oligonucleotide sequence that 


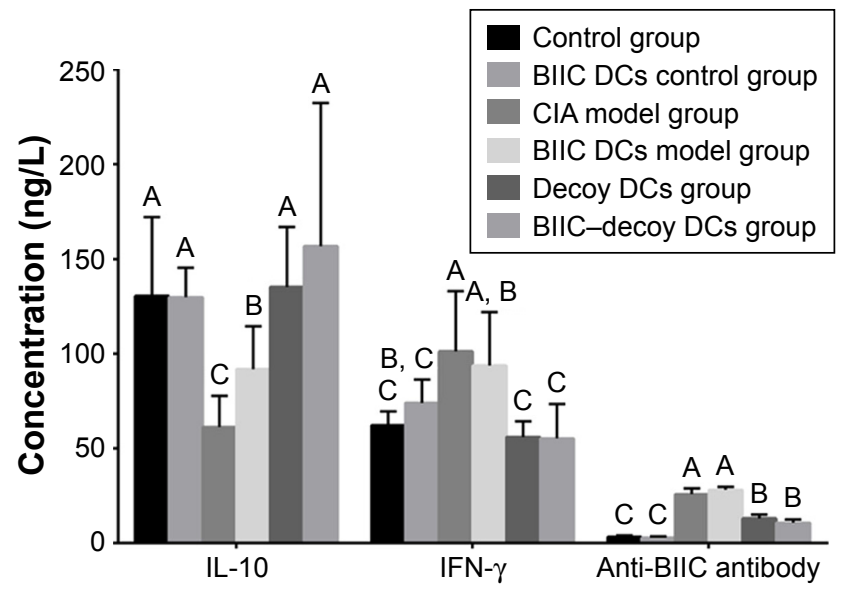

Figure 5 Effect of BIIC-decoy DCs on the serum levels of IFN- $\gamma$, IL-I0, and antiBIIC antibody in differently treated rats.

Notes: $n=10$. Different letters represent groups with extremely significant difference $(P<0.01)$.

Abbreviations: BIIC, bovine type II collagen; CIA, collagen-induced arthritis; DCs, dendritic cells.

is consistent with NF- $\mathrm{KB}$-specific binding sites, because of which NF- $\mathrm{KB}$ decoy ODNs can inhibit the transcription factor from regulating the expression of the target gene after their

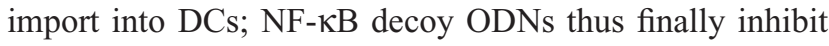
the phenotype and function of DC maturation and produce tDCs. ${ }^{18}$ tDCs express low levels of CD80 and CD86 and play an important role in immune and autoimmune tolerance by inhibiting T-cell response or by inducing apoptosis. ${ }^{19}$ The results from our study showed that NF- $\mathrm{KB}$ decoy ODNmodified DCs could effectively reduce the expression of CD80 and CD86 on the surface of rat spleen-derived DCs and thus inhibit T-lymphocyte proliferation, which indicated that imDCs were induced and tDCs were obtained.

The therapeutic effects of modified DCs have been studied in many immunological diseases. In mice, imDCs have been obtained by treating DCs with electric eel acetylcholine receptor immune advantage peptides (Talpha146-162) and these Talpha146-162-imDCs have been shown to inhibit the immune response of TAChR presensitized $\mathrm{T}$ cells and prevent the occurrence of experimental autoimmune myasthenia gravis. ${ }^{20}$ In addition, Yang et al showed that tDCs expressing high levels of IL-10 and low levels of IL-12 can induce antigen-specific tolerance in experimental autoimmune

Table I The serum level of antibody for human serum in rats (titer)

\begin{tabular}{lll}
\hline Groups & Rats $(\mathbf{n})$ & Titer \\
\hline BIIC-decoy DCs group & 5 & $\mathrm{I}: 2$ \\
Decoy DCs group & 5 & 0 \\
BIIC DCs model group & 5 & $\mathrm{I}: 4$ \\
\hline
\end{tabular}

Abbreviations: BIIC, bovine type II collagen; DCs, dendritic cells.

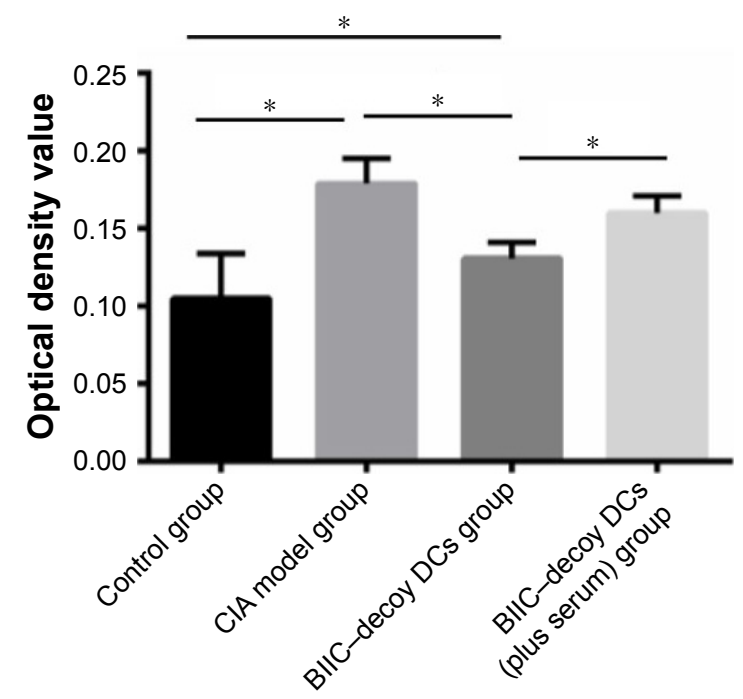

Figure 6 Proliferation degree of spleen cells in rats undergoing different treatments. Notes: $\mathrm{n}=10 ; * \mathrm{P}<0.05$

Abbreviations: BIIC, bovine type II collagen; CIA, collagen-induced arthritis; DCs, dendritic cells.

encephalomyelitis. ${ }^{21}$ Moreover, mouse bone marrow-derived DCs modified using Fas ligand can inhibit the progression of established CIA in DBA/1 mice by suppressing IFN- $\gamma$ production and reducing T-cell proliferation. ${ }^{22}$ Moreover, engineered bone marrow-derived DCs expressing IL-4 have been shown to inhibit Th1 responses and decrease the incidence and severity of CIA. ${ }^{23}$ In this study, we modified DCs using NF- $\kappa \mathrm{B}$ decoy ODNs and loaded them with a specific antigen and then tested their ability to regulate CIA. We treated CIA rats with tDCs loaded with a specific antigen (BIIC-decoy DCs), tDCs (decoy DCs), or nontolerance DCs loaded with a specific antigen (BIIC DCs) and found that the BIIC DCs failed to ameliorate arthritis, which indicated that mDCs loaded with a specific antigen could not repair the impaired immune response. However, the BIIC-decoy DCs, similar to the decoy DCs, inhibited immune reactions, reduced the serum level of IFN- $\gamma$ and anti-BIIC antibody, and increased IL-10 levels, finally alleviating the arthritis. This result indicated that the NF- $\mathrm{\kappa B}$ decoy ODN-induced tDCs loaded with a specific antigen could effectively inhibit CIA, possibly by regulating IFN- $\gamma$ and IL- 10 levels to balance the Th1/Th2 cytokines.

To evaluate the influence of these treatments on the body, the ability of the treated rats to regulate immune responses against unrelated antigens was assessed after immunization using human whole serum. Antibodies against whole human serum could be detected in the serum of the CIA rats injected with BIIC DCs or BIIC-decoy DCs but not in the rats treated with decoy DCs. This result indicated that tDCs loaded without a specific antigen may cause a nonantigen-specific and 
extensive immune suppression, while those exposed to the specific antigen (BIIC) can establish an antigen-specific immune tolerance and have no effect on unrelated antigen response. In addition, the BIIC-decoy DCs significantly reduced lymphocyte proliferation in the CIA rats and treatment with human serum (unrelated antigen) could recover the lymphocyte proliferation in these. These data showed that BIIC-decoy DCs could suppress the lymphocyte proliferation induced by the specific antigen (BIIC) without affecting lymphocyte proliferation provoked by other unrelated antigens. These results support the feasibility of arthritis treatment using NF- $\kappa$ B decoy ODN-modified DCs loaded with the specific antigen.

\section{Conclusion}

Our results suggest that NF- $\kappa \mathrm{B}$ decoy ODNs can induce spleen-derived DC immaturation and generate tDCs. Furthermore, the NF- $\kappa \mathrm{B}$ decoy ODN-induced tDCs loaded with BIIC could effectively alleviate CIA in rats, possibly by regulating the balance of Th1/Th2 cytokines and inhibiting T-cell activation and differentiation. Moreover, BIIC-decoy DCs could induce an antigen-specific immune tolerance without inducing antibodies against unrelated antigens. Therefore, we believe that NF- $\mathrm{KB}$ decoy ODN-modified DCs loaded with the specific antigen might be a new feasible approach for the treatment of autoimmune diseases in the future.

\section{Acknowledgments}

This study was supported by a district science fund of the National Natural Sciencse Foundation of China (grant number 81660361) and joint funds from the Guiyang Technology Bureau and Guizhou Medical University (grant number GY2015-38).

\section{Disclosure}

The authors report no conflicts of interest in this work.

\section{References}

1. Imperato AK, Bingham CO 3rd, Abramson SB. Overview of benefit/ risk of biological agents. Clin Exp Rheumatol. 2004;22(5 suppl 35): S108-S114.

2. Flores-Borja F, Mauri C, Ehrenstein MR. Restoring the balance: harnessing regulatory $\mathrm{T}$ cells for therapy in rheumatoid arthritis. Eur $J$ Immunol. 2008;38(4):934-937.

3. Cope AP, Schulze-Koops H, Aringer M. The central role of T cells in rheumatoid arthritis. Clin Exp Rheumatol. 2007;25(5 suppl 46):S4-S11.

4. Digre A, Singh K, Abrink M, et al. Overexpression of heparanase enhances $\mathrm{T}$ lymphocyte activities and intensifies the inflammatory response in a model of murine rheumatoid arthritis. Sci Rep. 2017;7:46229.
5. Tripathy A, Khanna S, Padhan P, Smita S, Raghav S, Gupta B. Direct recognition of LPS drive TLR4 expressing CD8+ T cell activation in patients with rheumatoid arthritis. Sci Rep. 2017;7(1):933.

6. Dhodapkar MV, Steinman RM, Krasovsky J, Munz C, Bhardwaj N. Antigen-specific inhibition of effector $\mathrm{T}$ cell function in humans after injection of immature dendritic cells. $J$ Exp Med. 2001;193(2): 233-238.

7. Lu L, McCaslin D, Starzl TE, Thomson AW. Bone marrow-derived dendritic cell progenitors (NLDC 145+, MHC class II+, B7-1dim, B7-2-) induce alloantigen-specific hyporesponsiveness in murine $\mathrm{T}$ lymphocytes. Transplantation. 1995;60(12):1539-1545.

8. Figdor CG, de Vries IJ, Lesterhuis WJ, Melief CJ. Dendritic cell immunotherapy: mapping the way. Nat Med. 2004;10(5):475-480.

9. Bonham CA, Peng L, Liang X, et al. Marked prolongation of cardiac allograft survival by dendritic cells genetically engineered with NF-kappa B oligodeoxyribonucleotide decoys and adenoviral vectors encoding CTLA4-Ig. J Immunol. 2002;169(6):3382-3391.

10. Giannoukakis N, Bonham CA, Qian S, et al. Prolongation of cardiac allograft survival using dendritic cells treated with NF- $\mathrm{KB}$ decoy oligodeoxyribonucleotides. Mol Ther. 2000;1(5 Pt 1):430-437.

11. Li R, Zhang Y, Zheng X, et al. Synergistic suppression of autoimmune arthritis through concurrent treatment with tolerogenic DC and MSC. Sci Rep. 2017;7:43188.

12. Xu MQ, Suo YP, Gong JP, Zhang MM, Yan LN. Prolongation of liver allograft survival by dendritic cells modified with NF-kappaB decoy oligodeoxynucleotides. World J Gastroenterol. 2004;10(16): 2361-2368.

13. Nouri-Shirazi M, Guinet E. Direct and indirect cross-tolerance of alloreactive $\mathrm{T}$ cells by dendritic cells retained in the immature stage. Transplantation. 2002;74(7):1035-1044.

14. Li Q, Verma IM. NF-kappaB regulation in the immune system. Nat Rev Immunol. 2002;2(10):725-734.

15. Guzman CA, Domann E, Rohde M, et al. Apoptosis of mouse dendritic cells is triggered by listeriolysin, the major virulence determinant of Listeria monocytogenes. Mol Microbiol. 1996;20(1):119-126.

16. Backstrom NF, Dahlgren UI. Induction of experimental arthritis in $\mathrm{BALB} / \mathrm{c}$ mice by inclusion of a foreign protein in the collagen inoculum. Scand J Immunol. 2008;67(4):322-328.

17. Gandy KL, Domen J, Aguila H, Weissman IL. CD8+TCR+ and CD8+TCR- cells in whole bone marrow facilitate the engraftment of hematopoietic stem cells across allogeneic barriers. Immunity. 1999; 11(5):579-590.

18. Vieira PL, de Jong EC, Wierenga EA, Kapsenberg ML, Kalinski P. Development of Th1-inducing capacity in myeloid dendritic cells requires environmental instruction. J Immunol. 2000;164(9):4507-4512.

19. O'Sullivan BJ, Thomas R. CD40 ligation conditions dendritic cell antigen-presenting function through sustained activation of NF-kappaB. J Immunol. 2002;168(11):5491-5498.

20. Wang R, Yang H, Xiao B, Zhang LF. [Effect of dendritic cells pulsed with Talpha146-162 on autoimmune myasthenia graves and the role of B cells activation]. Sichuan Da Xие Xие Bao Yi Xue Ban. 2009;40(5): 793-797. Chinese.

21. Yang JS, Xu LY, Huang YM, Van Der Meide PH, Link H, Xiao BG. Adherent dendritic cells expressing high levels of interleukin-10 and low levels of interleukin-12 induce antigen-specific tolerance to experimental autoimmune encephalomyelitis. Immunology. 2000;101(3):397-403.

22. Kim SH, Kim S, Oligino TJ, Robbins PD. Effective treatment of established mouse collagen-induced arthritis by systemic administration of dendritic cells genetically modified to express FasL. Mol Ther. 2002;6(5):584-590.

23. Morita Y, Yang J, Gupta R, et al. Dendritic cells genetically engineered to express IL-4 inhibit murine collagen-induced arthritis. J Clin Invest. 2001;107(10):1275-1284. 


\section{Publish your work in this journal}

Drug Design, Development and Therapy is an international, peerreviewed open-access journal that spans the spectrum of drug design and development through to clinical applications. Clinical outcomes, patient safety, and programs for the development and effective, safe, and sustained use of medicines are the features of the journal, which has also been accepted for indexing on PubMed Central. The manuscript management system is completely online and includes a very quick and fair peer-review system, which is all easy to use. Visit http://www.dovepress.com/testimonials.php to read real quotes from published authors.

Submit your manuscript here: http://www.dovepress.com/drug-design-development-and-therapy-journal 